Mots. Les langages du politique

$87 \mid 2008$

Chrononymes. La politisation du temps

\title{
Une démocratie illégitime. La década infame dans le discours populiste argentin
}

Erwan Sommerer

\section{(2) OpenEdition \\ Journals}

Édition électronique

URL : https://journals.openedition.org/mots/11962

DOI : $10.4000 /$ mots. 11962

ISSN : 1960-6001

Éditeur

ENS Éditions

Édition imprimée

Date de publication : 21 juillet 2008

Pagination : 27-41

ISBN : 978-2-271-06685-5

ISSN : 0243-6450

Référence électronique

Erwan Sommerer, « Une démocratie illégitime. La década infame dans le discours populiste argentin », Mots. Les langages du politique [En ligne], 87 | 2008, mis en ligne le 21 juillet 2010, consulté le 22 avril 2022. URL : http://journals.openedition.org/mots/11962; DOI : https://doi.org/10.4000/mots.11962 


\section{Une démocratie illégitime. La década infame dans le discours populiste argentin}

La década infame ("Décade infâme») est, dans l'histoire argentine, une période commencée et achevée par un coup d’État militaire : celui du général Uriburu en septembre 1930, et celui du Groupe des officiers unis (GOU) en juin 1943. Cette longue décennie est pour l'Argentine celle de tous les bouleversements économiques et sociaux, tandis qu'un régime démocratique instable et imparfait - basé sur la fraude et le clientélisme - se met en place. Surtout, à cette période succède une autre décennie cruciale, dominée par la figure de Juan Perón, leader populiste et autoritaire qui gouverne jusqu'en septembre 1955 en gagnant le soutien des populations ouvrières. Encore aujourd'hui, ces deux décennies sont perçues de façons très différentes : la première est reléguée dans l'ombre de l'âge d'or qu'aurait été la seconde. Ainsi, le chrononyme década infame marque une rupture entre un avant et un après fondés sur un contraste fort qui touche aussi à la pratique argentine de la démocratie. Cet avant et cet après sont une construction discursive : ils relèvent d'une interprétation imposée à l'issue d'un rapport de force qui s'est joué à la fois sur la place publique - entre des options partisanes concurrentielles - et dans la pensée politique.

Le mécanisme central de cette interprétation est concentré dans la désignation même de década infame : les acteurs principaux de cette période, son régime politique, sa situation sociale et économique, sont présentés comme autant d'antithèses radicales de la période suivante, celle du triomphe de Perón. Cet effet de contraste et de renversement agit comme un miroir : le pire de la década infame aurait été inversé pour faire place au meilleur du régime péroniste; tout ce qui faisait le caractère dramatique des années trente aurait été remplacé par son contraire. Mais cette interprétation en termes de basculement est, dans plusieurs domaines, en contradiction avec la ressemblance des deux périodes successives : la notion de década infame tend à masquer la proximité au profit de l'idée d'une rupture radicale. Et si la thèse du contraste est demeurée dominante, ce qu'indique la persistance actuelle de l'expression, c'est parce qu'elle a participé à la structuration d'un échiquier politique où la mythologie péroniste - encore centrale aujourd'hui - tient le premier rôle.

Université d'Aix-en-Provence, IEP, CREALC, erwan.sommerer@hotmail.fr 
Dès la fin des années soixante, la mobilisation étudiante et syndicale en faveur du retour au pouvoir de Perón s'est nourrie d'une narration historique qui avait une double fonction : l'une de fondation - la construction du mythe de l'âge d'or péroniste - et l'autre de réitération - la lutte pour le retour du leader populiste. Dès lors, comprendre la construction et le rôle de cette narration impose d'interroger d'abord le rapport complexe de distance et de proximité qui caractérise les deux périodes évoquées. Cela permet de mieux saisir comment, après 1955, a pu être réinterprétée l'évolution politique du pays, le régime péroniste devenant un paradis perdu. Analyser l'établissement d'une hiérarchie entre les deux périodes implique ensuite d'étudier les acteurs de l'énonciation et de la diffusion de cette mythologie et d'évoquer sa fonction dans le retour de Perón après dix-huit ans d'exil. Et sans qu'il soit question de réhabiliter la décennie que l'expression frappe d'indignité - ce fut une période très âpre à tous les points de vue -, il s'agit simplement de se demander si son discrédit au profit de la décennie péroniste ne participe pas aussi, sinon d'un même discrédit, du moins d'un déficit de légitimité de la démocratie représentative en Argentine.

\section{La década infame et la crise de la démocratie représentative}

\section{Les désignations de l'Argentine des années trente dans les études historiques}

Le syntagme década infame est souvent traduit en français par «décennie infâme », et en anglais par "infamous decade ». Renvoyant à la période des années trente, ce chrononyme couvre une plage temporelle de treize ans. Peu d'auteurs français (Béarn, 1975; Bourdé, 1987) ont utilisé "décade infâme», qui est pourtant la traduction la plus simple. À ce propos, on peut remarquer que ce choix - «décade» plutôt que «décennie»-, plutôt rare dans les travaux historiques français, apparait systématique chez Béarn, qui parle également de la "décade péroniste» en référence à la période 1946-1955 pendant laquelle Juan Perón fut président de l'Argentine². L'adjectif infame, pour

1. Le corpus utilisé pour cette analyse comporte vingt-sept ouvrages et articles en langues anglaise, espagnole et française. Ce sont des travaux d'historiens, de sociologues et de politistes dans lesquels les années trente sont évoquées, même lorsqu'elles ne sont pas étudiées en tant que telles. Dans ce corpus, quelle que soit la langue employée, on note une grande variation dans l'utilisation ou non d'une majuscule au début du chrononyme. De façon générale, toutefois, l'emploi d'une minuscule est plus courant en espagnol. En ce qui concerne le français, on a opté ici pour la traduction «Décade infâme ».

2. On considère souvent comme un anglicisme, donc comme impropre, l'utilisation de décade pour qualifier une période de dix ans et non de dix jours. Mais la racine du mot (deka en grec) renvoie simplement au sens de "dix» et n'impose donc aucun groupe spécifique (jours, années, etc). 
sa part, aurait pu être traduit également par «ignoble», voire «désastreuse». Mais la correspondance translinguistique la plus évidente est aussi celle qui permet de conserver le mieux l'impression d'indignité et de déshonneur attachée à cette période de l'histoire argentine; il s'agit, dans la mémoire historique, d'une décennie honteuse, marquée par ce qui a pu être considéré comme la «bassesse» de ses élites politiques. Quoi qu'on pense par ailleurs de la légitimité de cette désignation péjorative, force est donc de constater que la proximité linguistique entre l'espagnol, le français et, dans le cas présent, l'anglais ne recèle aucun piège sémantique pour le traducteur. Le consensus autour de la transposition quasi identique des termes s'appuie sur la très bonne conservation de la dimension dépréciative que revêt l'expression.

Toutefois, ce constat de la traductibilité assez aisée et spontanée de la signification attachée au syntagme ne change rien à l'essentiel du problème : le fait qu'il ne s'agit en aucun cas d'une dénomination neutre et objective, dépourvue de coloration partisane. Au contraire, ce terme est empreint d'une forte charge critique qui devrait dissuader les historiens et les politistes intéressés par l'Argentine - ou simplement évoquant cette période - de l'utiliser sans précautions. En effet, on le verra, la période des années trente a été nommée década infame par des acteurs du jeu politique argentin dont l'intérêt résidait précisément dans l'effet «repoussoir» contenu dans l'expression : il fallait ternir la période prépéroniste pour que l'Argentine de Perón en sortît par contraste auréolée d'une réputation d'opulence et de liberté, assimilée à l'âge d'or de la nation. Dans ce cas, utiliser década infame de façon neutre, banale, comme l'appellation normale de cette décennie, revient à accepter plus ou moins volontairement d'en ratifier le caractère partisan. Cela implique d'accepter le jugement disqualifiant que cette formulation produit et donc, dans une certaine mesure, d'accepter le point de vue polémique qui en est directement à l'origine. Un point de vue fondé sur une vision péroniste de l'histoire argentine, à laquelle un observateur scientifique n'est pas supposé adhérer de façon automatique, sans la discuter.

Or, parler sans plus de précautions de «Décade infâme», simplement parce que cette formule est entrée dans l'usage commun, c'est accepter d'inclure un élément idéologique dans des travaux qui, sans prétendre à l'idéal d'une neutralité absolue, doivent au moins faire preuve de distanciation envers de telles désignations péjoratives. Chez beaucoup de spécialistes de l'histoire argentine - qu'ils soient français, anglais ou espagnols -, cette distanciation est faible, voire minimale. Parfois, elle prend l'aspect de simples guillemets (Lancha, 2003; Norden, 1996; Walter, 1985 et 1993), qui peuvent être pour l'auteur un moyen discret de montrer qu'il ne souscrit pas totalement à la neutralité présumée de l'expression, et qu'il ne fait que rapporter une dénomination sur laquelle il ne se prononce pas explicitement. Cet effet de distanciation est en général plus marqué : c'est le cas lorsqu'il est question 
de «la période connue sous le nom de...» ou de «la période appelée...», modalisations autonymiques précautionneuses qu'on retrouve chez de nombreux auteurs 3 . La plupart des travaux historiques ne vont pas plus loin et, sauf exception (Rapoport, 1992 ; Sturzenegger, 2006), l'expression n'y est presque jamais discutée. Rares sont ceux où sont présentés d'autres syntagmes moins courants comme la « restauration conservatrice» (Quattrocchi-Woisson, 1993) ou «néo-conservatrice» (Buchrucker, 1987) : c'est le cas lorsque les auteurs, parce qu'ils s'intéressent aux discours partisans argentins, saisissent mieux l'appellation dans sa qualité de slogan issu d'une perspective idéologique. Mais dans la plupart des cas, la Décade infâme n'est pas abordée à travers cette problématique sémantique. Comme si qualifier d'infâme plus de dix ans de l'histoire argentine n'avait en fin de compte aucune conséquence notable.

Pour résumer, face à la formation d'une telle boite noire, on peut légitimement s'estimer insatisfait, et ce pour deux raisons. Omettre de remonter aux racines idéologiques du terme, c'est manquer sa fonction originelle dans la vie politique argentine : la notion stigmatisante a été diffusée par des militants de la gauche nationaliste et péroniste qui défendaient un projet de société étroitement lié au bilan politique et social des années trente. La formule est donc inscrite au départ dans un discours engagé, sans compter qu'elle est aujourd'hui très répandue, à la fois dans le sens commun argentin mais aussi - on l'a vu - dans les travaux de spécialistes du monde entier. Or cette banalisation, qui lui donne presque une apparence de «neutralité» malgré son sens péjoratif explicite, n'empêche nullement la préservation, sous forme certes atténuée, de sa dimension polémique. Dans un pays où les querelles historiographiques et idéologiques n'ont cessé de s'entremêler tout au long du $20^{\mathrm{e}}$ siècle, tomber - même de façon implicite ou discrète - dans le piège de la banalisation de l'expression revient à manquer à la fois la complexité de la décennie concernée et la construction sémantique dont elle a fait l’objet.

\section{De la «fraude patriotique » au populisme péroniste}

Comprendre l'origine et la fonction de l'expression década infame dans l'histoire politique argentine impose de connaitre les enjeux propres à cette époque. Les années trente furent pour les Argentins une période de difficultés à la fois économiques, politiques et identitaires. Le pays fut très durement touché par la crise de 1929 et connut une phase de recul de la démo-

3. C'est le cas de beaucoup de travaux historiques qui ne traitent pas spécifiquement de cette période, mais sont pourtant amenés à l'évoquer, même de façon ponctuelle. Par exemple : Bakewell, 2004; Balderston, Gonzales, 2004; Calveiro, 1988; De Luca, Jones, Tula, 2002; Gèze, Labrousse, 1975; Graham-Jones, 2000; Hora, 2001; Lewis, 2001; Mallorquín, 1998; Roniger, Sznajder, 1998; Smith, 1989; Ungar, 2002 ; Walter, 2000; West, 2001; Winn, 2006; Zanetta, 2004. 
cratie. Enfin, la décennie fut l'occasion d'une remise en cause profonde des éléments constitutifs de la nation argentine. En 1912, les conservateurs, issus de l'oligarchie agro-exportatrice, avaient accepté le principe du suffrage universel masculin et du vote secret, permettant aux classes moyennes d'accéder à la représentation politique. Le grand bénéficiaire de cette réforme fut le président Yrigoyen, issu de l'Union civique radicale (UCR), élu en 1916 puis en 19284. Mais son second mandat ne résista pas à la crise économique, et sa chute inaugura la Décade infâme. Ce fut d’abord le coup d’État du général Uriburu, soutenu par l'extrême droite (Buchrucker, 1987 ; Jackisch, 1997; Lafage, 1991 ; Rock, 1993). Puis, en novembre 1931, ce fut l'élection du général Justo à la tête de l'État. De fait, cela signifiait le retour au pouvoir de l'oligarchie conservatrice, reléguée depuis une quinzaine d’années dans les marges de la vie politique (Béarn, 1975).

La réforme électorale de 1912 ne fut pas abolie, mais la fraude et le clientélisme permirent aux conservateurs de se maintenir au pouvoir malgré un profond bouleversement du modèle économique argentin. En effet, après 1929, les revenus tirés de l'exportation du blé et de la viande - deux piliers du pouvoir oligarchique - s'effondrèrent, ce qui provoqua une montée rapide du chômage et un fort exode rural. Touchés par la misère, les chômeurs et les ouvriers formaient à la périphérie de Buenos Aires un prolétariat précaire, peu politisé, peu encadré par les syndicats (Bakewell, 2004; Béarn, 1975). L'oligarchie fit tout pour les maintenir à l'écart du système représentatif : Justo et son successeur furent élus en pratiquant ouvertement la «fraude patriotique »5. Selon eux, c'était aux seuls «vrais» Argentins, donc aux élites traditionnelles, de décider de l'avenir de la nation. La classe ouvrière était pour sa part constituée d'Argentins trop récents à leurs yeux, dont les parents espagnols, allemands et surtout italiens avaient émigré dans le pays au début du $20^{e}$ siècle. Face à ce discours élitiste, l'UCR se déchira en courants contradictoires : la présidence d'Yrigoyen - marquée par un style perçu comme autocratique et populiste avait provoqué de vifs débats internes. Mais l'UCR était aussi divisée sur la question de la participation aux élections frauduleuses. Alors qu'une frange du parti souhaitait renverser le régime par la force, la majorité choisit en 1935 la voie des urnes en espérant une libéralisation de la vie politique. Dans ce contexte, le coup d'État du 4 juin 1943 permit l'arrivée au pouvoir d'une junte

4. L'UCR a été fondée en juin 1891 en période de crise économique et sociale. Ses membres déclenchèrent plusieurs insurrections, notamment celle de 1905 menée par Yrigoyen. Dès l'origine, les revendications de l'UCR portaient sur la libéralisation du jeu politique à travers la réforme du mode de scrutin.

5. La «fraude patriotique» était une expression utilisée par les conservateurs eux-mêmes. Leur objectif était d'exclure du système électoral les populations jugées dangereuses pour l'Argentine, c'est-à-dire ne disposant pas selon eux du même attachement à la patrie. Par la suite, le discours péroniste inversa cette conception de la nation, érigeant les classes populaires au rang de seuls vrais Argentins face aux élites pro-occidentales. 
militaire disparate aux objectifs confus : elle voulait en finir avec l'agitation sociale, avec l'oligarchie archaïque et préserver la neutralité argentine dans le conflit mondial (Buchrucker, 1987; Lafage, 1991). Ce fut la fin d'une période complexe et équivoque, marquée par un régime représentatif tronqué (Rapoport, 1992). En gagnant plus tard le nom de década infame, elle prit une signification plus manichéenne puisée dans son opposition à la décennie suivante qui, de 1946 à 1955, vit la première ascension de Perón au pouvoir.

Le contraste entre les deux époques mérite à ce titre d'être interrogé. Qu'une rupture ait eu lieu ne saurait être nié; Perón fut l'initiateur d'un style de gouvernement qui procéda d'un changement d'attitude radical envers les classes populaires : il parvint au pouvoir avec le soutien d'une population que les élites traditionnelles et leurs adversaires de l'UCR avaient maintenue à l'écart du jeu politique, redessinant les contours de la nation argentine légitime. Ainsi, alors qu'en 1943 il n'était encore qu'un membre secondaire de la junte, il fit de l'alliance avec les syndicats le socle privilégié de ses ambitions. Il impulsa une politique sociale, réforma le droit syndical et le droit du travail, améliora les conditions de vie des ouvriers et obtint rapidement le soutien d'une part massive de l'électorat qui trouvait là un porte-parole inattendu (Buchrucker, 1987). Au cours des années suivantes, Perón consacra une grande partie des richesses nationales - l'Argentine exportait sa viande à prix d'or aux nations européennes en ruine - à cette clientèle électorale que nul n'avait auparavant osé mobiliser ainsi. À travers le dialogue prétendu exclusif entre le leader et son peuple, une autre conception de la démocratie était à l'œuvre : le système représentatif imparfait fut débordé par le populisme6. Et l'échiquier politique argentin subit dès lors une restructuration complète. Les clivages de la période antérieure s'estompèrent tandis que Perón faisait face à l'hostilité de la majorité des autres acteurs politiques. Face aux accusations de fascisme, il arguait de son rapport privilégié au peuple pour s'ériger en démocrate.

L'espace politique argentin bascula durablement avec sa victoire à la présidentielle de 1946, et ses adversaires furent relégués dans une opposition hétérogène et confuse. L'UCR, autrefois fer de lance de la libéralisation du régime, apparaissait comme une force archaïque et antidémocratique, attachée à un modèle politique, représentatif et libéral, que les aléas des années trente avaient discrédité. En 1946, l'élection fut exempte de fraude. Mais très vite, le régime devint plus autoritaire. L'espace d'expression dévolu à l'op-

6. De façon générale, le populisme latino-américain fut un mode d'insertion des classes ouvrières dans des espaces publics alors monopolisés par les oligarchies. Il s'appuya sur des stratégies de mobilisation partisane par lesquelles un leader ou un parti s'érigeait en porteparole privilégié du peuple, lui-même perçu comme un acteur homogène doté d'une volonté propre. Avec Perón, ses principaux représentants furent Getúlio Vargas au Brésil et Juan Velasco au Pérou (Laclau, 2005 ; Touraine, 1988). 
position s'amenuisa et, peu à peu, le caractère démocratique du régime se réduisit à la mise en scène du lien - prétendu direct et intuitif - de Perón et de son peuple. Ce fut la lente évolution du péronisme dans des pratiques qui rappelaient de plus en plus la décennie antérieure. Dès 1949, les exportations s'effondrèrent à nouveau; l'Europe n'avait plus besoin de la viande argentine. L'État s'endetta, ce qui força Perón à limiter sa politique sociale (Béarn, 1975). La répression s'accentua au fur et à mesure que le régime était contesté. En 1955, lorsque Perón fut renversé par un coup d'État militaire - qui fut appelé «Révolution libératrice»-, l'Argentine n'était ni plus riche, ni plus libre que lorsque l'oligarchie avait été chassée du pouvoir plus de dix ans auparavant. Et s'il y eut initialement une rupture entre la Décade infâme et la décennie péroniste, l'écart entre les deux périodes, du point de vue du fonctionnement réel des institutions démocratiques et du niveau de vie, s'était en fait considérablement amenuisé. Pourtant, ce n'est pas cette proximité que l'histoire argentine a retenue.

\section{Fixation et fonction d’une désignation disqualifiante}

\section{La réinterprétation de l'histoire par la Gauche nationale}

La chute de Perón en 1955 et sa fuite en exil - d'où il commence à organiser la lutte contre le nouveau régime - induit l'affrontement dans les domaines politique et culturel de deux conceptions antagonistes de l'histoire nationale. Les vainqueurs de Perón forment une coalition hétérogène où coexistent des nostalgiques de l'Argentine agro-exportatrice et des libéraux décidés à promouvoir une démocratie à l'occidentale. Leur bilan de l'expérience péroniste, vécue dans l'opposition, est impitoyable : ils considèrent leur «Révolution libératrice» comme la victoire contre la démagogie et l'autoritarisme populistes. Face à eux, une autre perception est à l'œuvre. Dans les rangs des péronistes dispersés, surtout au sein des syndicats qui restent fidèles à leur leader, le retour des élites traditionnelles apparait comme une régression par rapport à ce qui devient pour eux l'âge d'or de la période 1946-1955. Ce sont deux conceptions radicalement inverses de l'histoire politique récente du pays. Dans chaque camp, des idéologues publient des arguments en faveur de l'une ou l'autre des interprétations en conflit. Très vite, cependant, c'est la nostalgie envers la période péroniste qui se généralise, la désignation década infame étant constitutive de ce phénomène.

Le terme a été employé une première fois par un journaliste nationaliste, José Luis Torres, dans un livre virulent paru en 1945 et intitulé La década infame. L'oligarchie des années trente y est dénoncée pour ses liens avec «l'impérialisme anglo-saxon». Surtout, c'est la «fraude patriotique», dénoncée 
comme antidémocratique, que l'auteur s'attache à blâmer. Puis on rencontre l'expression dans des travaux historiques comme celui de José Luis Moreno, Las Ideas políticas en Argentina (1946). Mais il faut attendre la chute de Perón pour que le syntagme se retrouve au cœur de plusieurs livres à succès. En 1960 parait La formación de la conciencia nacional de Juan José Hernandez Arregui7, qui comporte un long chapitre charnière consacré à la période des années trente, intitulé : Forja y la lucha popular por la liberación nacional (la «década infame»). Deux ans plus tard (1962) est publié Forja y la década infame par Arturo Jauretche ${ }^{8}$, qui prolonge sa réflexion en 1966 dans un nouveau livre, El medio pelo en la Sociedad Argentina. Dans ces trois essais d'analyse historique et politique, la Décade infâme est décrite sous un angle très critique. Les auteurs placent au centre de leur démonstration une organisation politique, la Force d'orientation radicale de la jeunesse argentine (Forja), qui a existé entre juin 1935 et octobre 1945, date à laquelle elle s'est dissoute à l'intérieur du mouvement péroniste triomphant.

Chez les auteurs concernés, la présentation du rôle de la Forja dans l'histoire argentine est essentielle à la compréhension de la Décade infâme et de ses suites. Chez eux, l'expression est quasi automatiquement associée à la description de ce groupe politique. La Forja, en effet, était issue d'une branche dissidente de l'UCR qui refusait la participation au jeu électoral truqué (Sigal, 1996). Ses membres se réclamaient d'un nationalisme de gauche, contestataire et souverainiste, visant à donner au peuple argentin la possibilité de s'exprimer par-delà les mécanismes représentatifs (Buchrucker, 1987). Ils critiquaient l'impérialisme anglais, décrivant l'Argentine comme une colonie aux mains d'une oligarchie vendue à l'étranger. Leur leader était Arturo Jauretche qui, plus de vingt plus tard, revient sur cette expérience et lui donne une logique historique : la Décade infâme est pour lui une période de soumission aux intérêts étrangers, lorsqu'un peuple réduit au silence a subi la domination frauduleuse de partis hostiles à la nation véritable. La Forja ranimait dans ce contexte ce qu'Arregui appelle la «conscience nationale», c'est-àdire la défense des intérêts de la nation, incarnée par le peuple. Pour ces deux auteurs, le rôle de la Forja fut de dévoiler les ressorts de la Décade infâme. Ce faisant, elle annonçait selon eux le péronisme qui, effectivement, se fit l'écho d'une partie de ses idées dans le populisme d’État. Mais cette interprétation est une construction ex post : la Forja fut peu influente sur le plan politique,

7. Juan José Hernandez Arregui (1913-1974) était un intellectuel argentin qui oscilla toute sa vie entre marxisme et péronisme. Il fut un théoricien de ce qu’il appelait lui-même la Gauche nationale argentine.

8. Arturo Jauretche (1901-1974) fut l'un des intellectuels argentins les plus en vue de son époque. Sous le régime péroniste, il fut nommé président de la banque de la province de Buenos Aires et dut s'exiler après la chute de Perón en 1955. Il ne cessa ensuite de publier des essais politiques. Dans les années soixante-dix, il prit position en faveur de la jeunesse péroniste, qui formait l'aile gauche du mouvement. 
et ses membres n'occupèrent que des fonctions subalternes sous le régime péroniste (Galasso, 1985, 1986). La construction d'une filiation idéologique entre les forjistes - transformés pour l'occasion en prophètes - et Perón est donc une réinterprétation de l'histoire argentine9, menée cependant à partir d'une convergence effective vingt ans plus tard.

Au début des années soixante, en effet, Perón est en exil. Devant la nécessité de mobiliser ses partisans, son discours se durcit et prend des inflexions de plus en plus révolutionnaires. Dans ce cadre, des sympathisants comme Jauretche et Arregui peuvent offrir à la doctrine péroniste une interprétation historique valorisante destinée à renforcer un discours populiste demeuré jusque-là pragmatique. Ainsi, ils inscrivent Perón dans un vaste tableau historique où domine le conflit entre le peuple et les forces impérialistes. La Forja et Perón sont alors les incarnations successives du camp «populaire», tandis que la Décade infâme devient le moment d'apogée du colonialisme antidémocratique. Dansles livres évoqués, letermeestassociéauxaspectsles plus négatifs des années trente : la domination étrangère et la perte de souveraineté, la corruption et la fraude, c'est-à-dire la captation de la démocratie sous couvert de libéralisme. La désignation década infame renvoie aux registres de la trahison, de la falsification et de l'usurpation : les élites «colonisées » sont déconnectées du peuple; elles aliènent la nation en la privant de son identité propre. Arregui évoque « une époque d'humiliation » caractérisée par «l'échec de la démocratie libérale, la fraude oligarchique et l'abandon du pays à l'impérialisme britannique» (p. 10) et Jauretche dénonce en 1966 cette «infamie» que fut « la trahison délibérée et consciente de la destinée du pays» (p.48).

Les thèmes initialement développés par Torres sont donc repris dans une interprétation plus ample et mis au service d'une description - par contraste bien plus positive de la décennie péroniste. À ce titre, la force de l'appellation disqualifiante, son lien au déshonneur et à la bassesse, insèrent les années trente dans un tableau sinistre dont le péronisme est alors l'envers absolu: l'expérience péroniste fut la première à offrir au peuple la possibilité de s'exprimer. Les difficultés du début des années cinquante ne sont pas passées sous silence, mais à l'aune de la Décade infâme, elles sont relativisées : Perón a pu hésiter ou se tromper, mais sa cause était juste. Le registre est cette fois celui de la «restauration» de la nation, de la prise de parole par le peuple, de la réalisation d'une attente et d'un manque enfin comblé. La victoire péroniste de 1946 apparait comme une libération porteuse de tout de ce qui faisait défaut: la démocratie réelle, l'indépendance nationale, la moralité de l'État et l'opulence. Dans les livres de Jauretche et Arregui, la Décade infâme acquiert

9. Dans son livre de 1946 sur les idées politiques en Argentine, l'historien José Luis Romero ne distingue pas de lien entre la Forja et le péronisme : alors que Perón est à ses yeux une incarnation argentine du fascisme européen, il présente les forjistes comme des intellectuels ayant cherché vainement à défendre la démocratie en période de fraude électorale (Romero, 1946). 
son sens en opposition aux idéaux de la Forja, qui annoncent pour leur part l'âge d'or du péronisme.

\section{Les relais universitaires et politiques}

Les livres de Jauretche et Arregui marquent le moment clé de réappropriation de l'expression década infame au service d'un projet nationaliste et souverainiste qui se tient à la croisée du péronisme et du socialisme révolutionnaire. Ce projet est idéologique dans le sens où il vise, durant l'exil de Perón, à diffuser une interprétation de l'histoire argentine qui permette au leader populiste de conserver sa popularité et de préparer son retour. Rapidement, certains universitaires adoptent l'expression et s'intéressent au rôle de la Forja. C'est le cas dans le classique Partidos y poder en la Argentina d'Alberto Ciria, réédité plusieurs fois et traduit en anglais (Ciria, 1964). Jauretche et Arregui y sont cités et, si le rôle des forjistes est discuté et nuancé, la description de la Décade infâme révèle l'influence du nationalisme de gauche. Ciria, à ce titre, prépare la banalisation du terme dans les études historiques. Et tout cela participe au travail de mobilisation des partisans péronistes, leur fournissant un discours prêt à l'emploi : les auteurs concernés radicalisent l'image de Perón et en font un leader anti-impérialiste et révolutionnaire éloigné du Perón pragmatique et autoritaire de la période 1946-1955 (Rapoport, Spiguel, 1998). En fait, ils ne voient le péronisme que sous sa face contestataire, oubliant son aspect interclassiste et capitaliste. Ni Jauretche, ni Arregui n'occultent ces ambivalences, mais ils espèrent peser sur les choix du leader populiste. Pour cela, ils disposent d'un allié qui, pendant quelques années, est un proche de Perón. Il s’agit de John William Cooke, ex-député et représentant officiel de l'aile gauche du péronisme.

Cooke fait le lien entre Jauretche et Arregui, plus ancrés dans les milieux intellectuels, et Perón lui-même. C'est à la fois un militant et un théoricien influencé par le guévarisme (Galasso, 1997, Mazzeo, 1999). Après 1955, il joue un rôle crucial dans la réorganisation du mouvement péroniste clandestin. En novembre 1959, dans un discours intitulé La lucha por la liberación national (Cooke, 1971), prononcé à Buenos Aires devant une assemblée péroniste, il se réfère à la Décade infâme. Quelques années plus tard, il y consacre une partie de son livre Apuntes para la militancia (Cooke, 1964). À cette occasion, il associe de façon étroite les années trente à l'impérialisme anglais, à la «légalisation» de la fraude et à la trahison des élites politiques. L'arrivée de Perón au pouvoir est détaillée sous le titre «La brise de l'histoire »: à l'infamie succède l'air pur de la «vraie » démocratie (p. 21). En plus de la reprise du syntagme, on observe la même relation de basculement et de contraste que chez Jauretche et Arregui. La décennie péroniste serait le renversement parfait de la période précédente. Et il faut noter que l'on retrouve à la même époque une 
analyse semblable chez des historiens d'obédience marxiste qui, séduits par le nationalisme de gauche, apportent dès les années quarante un soutien critique au péronisme. C'est le cas notamment de Jorge Spilimbergo avec la publication de Nacionalismo oligárquico y nacionalismo revolucionario (1958) et surtout de Jorge Abelardo Ramos ${ }^{10}$ dans El Partido comunista en la política Argentina (1962) et dans son œuvre principale : Revolución y contrarevolución en la Argentina (1965). Sans être vraiment péronistes, et sans attacher autant d'importance à la Forja, ces auteurs participent à la diffusion de l'expression década infame - présente dans leurs livres - et interprètent de la même façon l'histoire argentine et la «Révolution libératrice» de 1955.

Pour tous, la chute de Perón a été le retour à la Décade infâme, tout comme la chute d'Yrigoyen avait signifié le retour à la période censitaire. La plupart des ex-forjistes et des partisans de la Gauche nationale ont perçu la défaite du péronisme comme une régression permettant à l'oligarchie de revenir au pouvoir. Jauretche, dans son livre de 1966, applique un schéma historique lapidaire à cette évolution : «1930 et 1955 sont des dates équivalentes, la Décade infâme et la révolution libératrice se rejoignent par leurs objectifs, par la technique révolutionnaire, par les équipes gouvernementales et par la même utilisation des forces armées destinées à jouer le rôle incroyable d'obstacle à la grandeur nationale» (p. 178). Ce qui importe ici est l'effet de réitération. Pour Jauretche, 1955 est le recommencement de la Décade infâme, et seul le retour de Perón doit permettre de restaurer la démocratie « réelle» : l'interprétation partisane du passé commande sa réitération cyclique. Chez Jauretche, Arregui ou Cooke, comme chez Spilimbergo et Abelardo Ramos, l'histoire argentine s'articule autour de la répétition d'un unique conflit entre le peuple et les élites pro-impérialistes. La Décade infâme et son dépassement par le péronisme furent des moments d'exacerbation d'un conflit dont ils n'étaient qu'une expression conjoncturelle. Perón prolongeait Yrigoyen, qui lui-même prolongeait Rosas ${ }^{11}$ (Quattrocchi-Woisson, 1993), et ainsi de suite. Pour les auteurs cités, l'important était alors que l'histoire se répète au moins une fois encore.

Ce qui, dans les années soixante, est nommé década infame est donc double. Il s'agit tout autant d'une époque précise, passée au filtre de la grille

10. Jorge Abelardo Ramos (1921-1994) fut un historien et un militant nationaliste influencé par le trotskisme, partisan d'une "gauche nationale » capable de rompre avec le statut semi-colonial de l'Argentine. Il analysa les aspects bonapartistes du péronisme, tout en animant un courant politique destiné à tenter de capter la dynamique révolutionnaire initiée par le populisme de Perón, qu'il soutint de façon stratégique.

11. Juan Manuel de Rosas, caudillo du 19e siècle opposé aux républicains et à leurs alliés anglosaxons, gouverna l'Argentine entre 1835 et 1852 . Longtemps considéré comme un tyran, il fit l'objet d'une réhabilitation par des historiens nationalistes qui en firent, finalement, un précurseur anti-impérialiste de Perón. Encore aujourd'hui, il demeure une figure controversée de l'histoire argentine. 
de lecture péroniste, que d'un terme plus général extensible à l'ensemble de l'histoire argentine. Toute époque de trahison du peuple, de recul démocratique et de domination «impérialiste» est vouée à être une répétition de la Décade infâme ${ }^{12}$. Cet effet de réitération est crucial : dans le contexte des années soixante, déprécier les années trente, nourrir la nostalgie de la décennie péroniste, revient à militer pour le retour de Perón et à promettre un nouvel âge d'or. Cette construction historique devient alors largement populaire, et la désignation péjorative de la période concernée fait l'objet d'un consensus : en effet, elle sert aussi les intérêts de Frondizi, président de 1958 à 1962, qui tente vainement de contrer la popularité de Perón par une politique développementaliste (Donghi, 1999; Sigal, 1996). Son échec, sous la pression de l'armée et des syndicats péronistes, fait de Perón le seul bénéficiaire de cette réécriture de l'histoire articulée autour du contraste entre la Décade infâme et la décennie suivante, érigée à un rang quasi mythologique ${ }^{13}$. Et c'est finalement la réception de cette narration historique par une nouvelle génération d'étudiants fascinés par la révolution cubaine qui, à la fin des années soixante, s'avère décisive : imprégnés des idées de la Gauche nationale, les Montoneros - guérilleros péronistes et marxistes jouent un rôle clé dans le retour, en octobre 1973, d'un Perón qu'ils confondent avec un Castro argentin (Gillespie, 1982 ; Sigal, Verón, 1986). La dévalorisation durable des années trente a permis d'occulter efficacement les aléas du péronisme originel au profit d'un mythe mobilisateur lié à la mémoire d'un âge d'or anti-impérialiste.

Le chrononyme década infame est né dans les milieux nationalistes pour désigner une période définie par trois éléments : la captation des institutions par l'élite agro-exportatrice déstabilisée par la crise de 1929, le dysfonctionnement du régime représentatif et la pression diplomatique du Royaume-Uni. Ces éléments ne sauraient être réfutés. Mais à cette réalité a été apposée une désignation destinée à en exacerber les aspects négatifs. On l'a vu, l'expression est étroitement liée à un discours anti-impérialiste et souverainiste dont les multiples théoriciens de la Gauche nationale furent les énonciateurs privilégiés. Mis au service du populisme péroniste, ce discours fut l'une des

12. On peut ainsi constater qu'en Argentine, les années quatre-vingt-dix, marquées par la politique néo-libérale du président Menem, sont régulièrement présentées comme une nouvelle Décade infâme, au cours de laquelle la souveraineté du pays aurait subi l'influence d'un modèle économique exogène et inapproprié (Grassi, 2003). En octobre 2004, le président Kirchner a ainsi qualifié cette période de «seconde Décade infâme».

13. Si l'on suit l'argumentation convaincante de Donghi sur ce point, on pourrait presque avancer l'idée que, sous Frondizi, le consensus autour de la signification des années trente comme Décade infâme est établi, et que le conflit ne se déroule plus tant au niveau idéologique (sur le sens) que politique : il s'agit simplement de déterminer quel leader - Frondizi ou Perón - exploitera le mieux les bénéfices du consensus. Perón, en tant que personnage intégré à la narration historique elle-même, était à ce titre dans une position plus avantageuse. 
sources de la contestation politique qui permit le retour au pouvoir de Perón. Mais le discrédit des années trente était donc aussi celui d'un régime. L'installation durable du péronisme sur l'échiquier politique argentin, l'aura positive dont la mémoire de Perón bénéficie encore aujourd'hui, sont les marques d'un choix qui a été fait entre deux perceptions de la démocratie : entre le libéralisme de l'UCR, et une version populiste où la mobilisation du peuple, même fictive et incantatoire, est préférée aux ambigüités du mandat représentatif. La légitimité et la validité du modèle populiste n'ont pas à être discutées ici. Mais l'on peut toutefois s'interroger sur le rôle du chrononyme dans la domination de ce modèle depuis des décennies.

Ainsi, en mai 2003, c'est un péroniste de gauche, Nestor Kirchner, qui a été élu alors que le pays venait de vivre l'effondrement de son économie. Son discours se basait sur l'appel au peuple et à la justice sociale, dénonçant le FMI, l'ennemi extérieur. Jamais le péronisme, malgré ses écueils, n'a été discrédité; les années quarante - incarnées aussi par Evita ${ }^{14}$ - demeurent un âge d'or. Et la Décade infâme est toujours la décennie maudite à laquelle sont renvoyés ceux qui défendent une autre conception - plus représentative et libérale - de la démocratie. Les rares victoires de l'UCR - celle de Raúl Alfonsín jusqu'en 1989 puis celle de Fernando de la Rúa en 1999 - se sont achevées dans une tourmente politique et sociale dont le péronisme est toujours sorti vainqueur. Bien sûr, l'expression a perdu une partie de son ancrage idéologique. De même, son emploi dans un texte n'implique pas une adhésion sans réserve au péronisme. Mais, d'une certaine manière, sa banalisation reste liée à la persistance dans l'espace public argentin d'un discours populiste pour lequel la relation au peuple doit passer avant les mécanismes moins spectaculaires du système représentatif. Derrière une simple expression se profile donc la hiérarchisation d'évènements historiques dont le souvenir structure encore la vie politique du pays.

\section{Références}

ARREgUI Juan José Hernández, 1960, La formación de la conciencia nacional, Buenos Aires, Plus Ultra.

BAKEWELL Peter, 2004, A history of Latin America, Oxford, Blackwell Publishing.

Balderston Daniel, Gonzales Mike, 2004, Encyclopedia of Latin American and Caribbean Literature, Oxford, Routledge.

BÉARN Georges, 1975, La Décade péroniste, Paris, Gallimard / Julliard.

14. Eva Perón, surnommée «Evita» (1919-1952), était la femme de Juan Perón. Elle participa à la mise en place du populisme d'État - notamment en organisant la branche féminine du péronisme. Ses origines modestes, sa popularité auprès des descamisados (les "sans-chemises", base militante de Perón) et sa mort prématurée à l'âge de 33 ans en firent dès les années soixante l'un des symboles de l'âge d'or péroniste. 
BOURDE Guy, 1987, La classe ouvrière argentine, 1929-1969, Paris, L'Harmattan.

BUCHRUCKER

Cristián, 1987, Nacionalismo y peronismo, Buenos Aires, Editorial Sudamericana.

CALVEIRo Pilar, 1988, «Syndicatosy Politica (Argentina 1980-1986)», Trujillo Bolio M. éd., 1988, Organización y luchas del movimiento Obrero Latinoamericano, 1978-1987, Buenos Aires, Editoria Siglo xxI.

COOKE John William, 1964, Apuntes para la militancia, Buenos Aires, Schapire Editor.

- 1971 [1959], La lucha por la liberación national, Buenos Aires, Ediciones Papiro.

De LuCA Miguel, Jones Marc P., Tula Maria Inès, 2002, «Buenos Aires, The evolution of local governance», D. Myers, H. Dietz éd., Capital City Politics in Latin America Democratization and Empowerment, Boulder, Lynne Rienner Publishers.

DoNGHI Tulio Halperin, 1999, «Argentin ponder the burden of the past», J. Adelman éd., Colonial Legacies, Oxford, Routledge.

GALASSO Norberto, 1985, Raul Scalabrini Ortiz, Buenos Aires, Ediciones del Pensamiento Nacional.

- 1986, J.-J. Hernández Arregui, Buenos Aires, Ediciones del Pensamiento Nacional.

- 1997, Cooke: de Perón al Che, Buenos Aires, Ediciones Homo Sapiens.

GÈze François, LABrousSE Alain, 1975, Argentine, révolution et contre-révolutions, Paris, Le Seuil.

GILleSPIE Richard, 1982, Soldados de Perón, Buenos Aires, Grijalbo.

Graham-Jones Jean, 2000, Exorcising History, Argentine Theater Under Dictatorship, Lewisburg, Bucknell University Press.

GrASSI Estela, 2003, Políticas y problemas sociales en la sociedad neoliberal : la otra década infame, 2 vol., Buenos Aires, Espacio.

Hora Roy, 2001, The Landowners of the Argentine Pampas. A Social and Political History, 1860-1945, Oxford, Oxford University Press.

JACKISCH Carlota, 1997, El nazismo y los refugiados alemanes en la Argentina, Buenos Aires, Editorial de Belgrano.

JAURETCHE Arturo, 1962, Forja y la década infame, Buenos Aires, Editorial Coyoacán.

- 1966, El medio pelo en la Sociedad Argentina, Buenos Aires, A. Peña Lillo Editor.

LACLAU Ernesto, 2005, On Populist Reason, Londres, Verso.

LAFAGE Franck, 1991, L'argentine des dictatures, Paris, L'Harmattan.

LANCHA Charles, 2003, Histoire de l'Amérique hispanique de Bolívar à nos jours, Paris, L'Harmattan.

LEWIS Daniel K., 2001, The History of Argentina, Wesport, Greenwood Press.

Mallorquín Carlos, 1998, Ideas e historia en torno al pensamiento económico latinoamericano, México, Editorial Plaza y Valdes.

Mazzeo Miguel éd., 1999, Cooke, de Vuelta, Buenos Aires, Ediciones La Rosa Blindada.

Norden Deborah Lee, 1996, Military Rebellion in Argentina, Lincoln, University of Nebraska Press.

QUATTROCCHI-WoIsson Diana, 1993, Un nationalisme de déracinés, Paris, CNRS.

Ramos Jorge Abelardo, 1962, El Partido comunista en la política Argentina, Buenos Aires, Coyoacan.

- 1965, Revolución y contra-revolución en la Argentina, Buenos Aires, Plus Ultra. 
RAPOPORT Mario et SPIguel Claudio, 1998, Les États-Unis et l'Argentine de Perón, Paris, L'Harmattan.

RAPOPORT Mario, 1992, "Argentina», L. Bethell, I. Roxborough éd., Latin America Between the Second World War and the Cold War, Cambridge, Cambridge University Press.

Rock David, 1993, Authoritarian Argentina, Berkeley, University of California Press.

Romero José Luis, 1946, Las ideas políticas en Argentina, Buenos Aires, Fondo de cultura económica.

Roniger Luis, Sznajder Mario, 1998, Constructing Collective Identities and Shaping Public Spheres, Latin American Paths, Eastbourne, Sussex Academic Press.

Sigal Sylvia, 1996, Le rôle politique des intellectuels en Amérique Latine, Paris, L'Harmattan.

Sigal Sylvia, Verón Eliseo, 1986, Péron o Muerte, Buenos Aires, Legasa.

SMITH William Charles, 1989, Authoritarianism and the Crisis of the Argentine Political Economy, Stanford, Stanford University Press.

SPILIMBERGO Jorge Enea, 1958, Nacionalismooligárquicoynacionalismorevolucionario, Buenos Aires, Editorial Amerindia.

Sturzenegger Odina, 2006, L'Argentine, Paris, Karthala.

Touraine Alain, 1988, La parole et le sang, Politique et société en Amérique Latine, Paris, Odile Jacob.

ToRres José-Luis, 1945, La década infame, Buenos Aires, Editorial de formación Patria.

UnGAR Mark, 2002, Elusive Reform Democracy and the Rule of Law in Latin America, Boulder, Lynne Rienner Publishers.

WALter Lynn, 2000, Women's Rights. A Global View, Wesport, Greenwood Press.

WALtER Richard J., 1985, The Province of Buenos Aires and Argentine Politics, 1912-1943, Cambridge, Cambridge University Press.

- 1993, Politics and Urban Growth in Buenos Aires, 1910-1942, Cambridge, Cambridge University Press.

WeSt Jacqueline, 2001, South America, Central America and the Carribean, Oxford, Routledge.

Winn Peter, 1999, Americas: The Changing Face of Latin America and the Caribbean, Berkeley, University of California Press.

ZANETTA Cecilia, 2004, The Influence of the World Bank on National Housing and Urban Policies, Londres, Ashgate Publishing. 